\title{
concept
}

\section{Theribosomeand YidC}

\section{New insights into the biogenesis of Escherichia coli inner membrane proteins}

\author{
Jan-Willem L. de Gier ${ }^{1+} \&$ Joen Luirink ${ }^{2}$ \\ ${ }^{1}$ Arrhenius Laboratories, Stockholm University, Stockholm, Sweden, and ${ }^{2}$ Vrije Universiteit, Amsterdam, The Netherlands
}

\begin{abstract}
In the bacterium Escherichia coli, inner membrane proteins (IMPs) are generally targeted through the signal recognition particle pathway to the Sec translocon, which is capable of both linear transport into the periplasm and lateral transport into the lipid bilayer. Lateral transport seems to be assisted by the IMP YidC. In this article, we discuss recent observations that point to a key role for the ribosome in IMP targeting and to the diverse roles of YidC in IMP assembly.

EM BO reports 4, 939-943 (2003)

doi:10.1038/sj.embor.embor921
\end{abstract}

\section{Introduction}

The Escherichia coli targeting factor signal recognition particle (SRP) consists of a 48-kDa subunit called fifty-four homologue (Ffh) and 4.5S RNA, which are homologous to the eukaryotic SRP54 and the 7S RNA, respectively (Herskovits et al., 2000). The latter two form part of the larger eukaryotic SRP, which functions in the cotranslational targeting of both membrane and secretory proteins, through the SRP receptor, to the Sec translocon in the membrane of the endoplasmic reticulum (ER).

The E. coli SRP is specialized in the co-translational targeting of inner membrane proteins (IM Ps), whereas secreted E. coli proteins follow a post-translational pathway that is mediated by the chaperone SecB (Herskovits et al., 2000). The SRP has been shown to interact specifically with the predominantly hydrophobic targeting signal at the amino termini of nascent IM Ps at an early stage in IM P biosynthesis, when the signal sequence is not yet fully exposed outside the ribosome (Ullers et al., 2003; Valent et al., 1997). Unlike its eukaryotic counterpart, the E. coli SRP is not thought to arrest translation after it binds to the targeting signal (Herskovits et al., 2000), although this view has recently been challenged

${ }^{1}$ Department of Biochemistry and Biophysics, Arrhenius Laboratories

Stockholm University, SE-106 91 Stockholm, Sweden

${ }^{2}$ Department of M icrobiology, VrijeUniversiteit, DeBoelelaan 1087 ,

1081 HV Amsterdam, The Netherlands

+Corresponding author. Tel: +468 162420; Fax: +46 8 153679;

E-mail: degier@dbb.su.se

Submitted 12 May 2003; accepted 21 July 2003
(Avdeeva et al., 2002). O nce formed, the ribosome-nascent chain (RNC)-SRP complex is targeted to the Sec translocon through a specific interaction between the SRP and its receptor, FtsY (Fig. 1C). In contrast to the membrane-bound mammalian SRP receptor, FtsY is only transiently associated with the plasma membrane and might contact the RNC-SRP complex in the cytoplasm (Herskovits et al., 2000). FtsY is also thought to deliver ribosomes to the membrane in an SRP-independent manner (H erskovits et al., 2001), and the subsequent synthesis of a membrane protein by these targeted ribosomes has been proposed to trigger SRP binding. Irrespective of the preceding steps, the transfer of the RNC to the Sec translocon is coordinated by the concerted action of Ffh and FtsY, both of which are GTPases, although the mechanistic details of this step are unclear.

In vitro crosslinking experiments suggest that in E. coli, the RN C s contact the SRP in the cytoplasm and then contact the Sectranslocon components when the nascent protein is approximately the same length (Ullers et al., 2003). This suggests that membrane insertion occurs co-translationally, as is the case in eukaryotes. Consistent with this model, the affinity of ribosomes for the Sec translocon is similar in E. coli and eukaryotic cells (Prinz et al., 2000). D uring IMP assembly, transmembrane domains (TM s) are arrested in the Sec translocon and subsequently move out into the lipid bilayer (Chen et al., 2002; De Gier \& Luirink, 2001). YidC, which is required for viability, is thought to assist in this transfer (De Gier \& Luirink, 2001). In addition, YidC mediates the membrane insertion of SRP/Sec-translocon-independent bacteriophage proteins (Fig. 1A; Chen et al., 2002).

Here, we discuss recent observations that point to a key role for the ribosome itself in IMP targeting and to diverse roles of YidC in IM P assembly.

A regulatory role for ribosomal proteins in IM P targeting? Recent evidence suggests that the ribosome has an important regulatory role at several stages of SRP-mediated targeting. The eukaryotic ribosomal subunits L23a and L35, which are close to the nascent-chain exit site, have been shown to constitute the ribosome attachment site for SRP54 (Pool et al., 2002). This strategically positions SRP54 to scan emerging polypeptides for the 


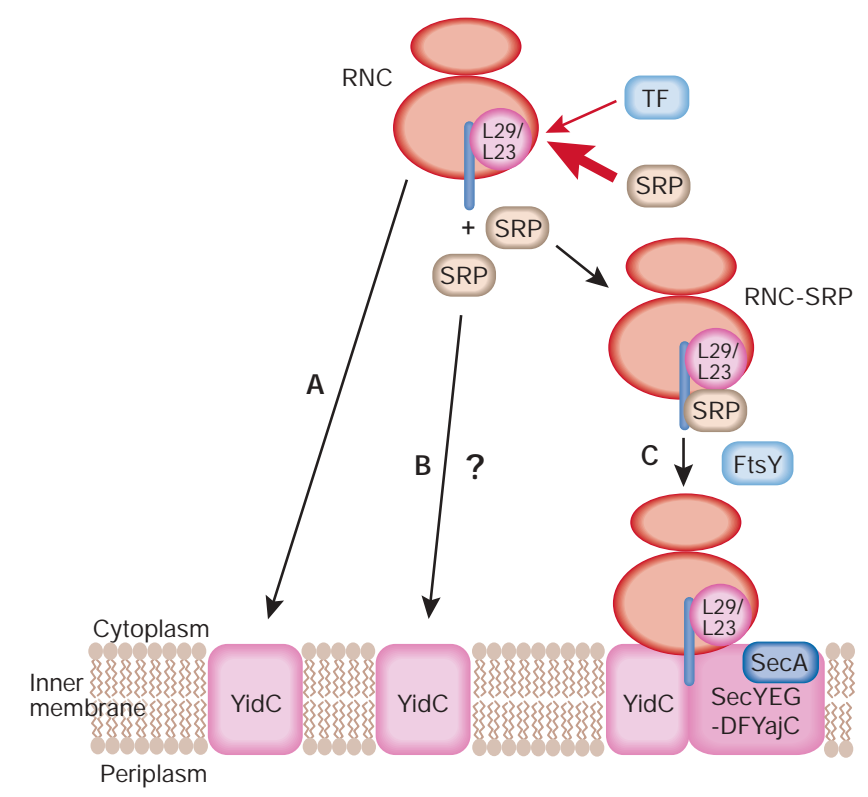

Fig. 1 | M odel of the roles that the ribosome and YidC might have in the targeting and insertion of membrane proteins in Escherichia coli. Two inner membrane protein (IM P) assembly pathways are known to exist in E. coli: (A) theYidC pathway and (C) the SRP-Sec translocon-YidC pathway. In addition, an SRP-YidC pathway (B) might be operational. The ribosome seems to have a key role in the targeting of at least SRP-Sec transloconYidC-dependent IM Ps. FtsY, SRP-receptor; L23, L23 component of the large subunit of the ribosome; $L 29, L 29$ component of the large subunit of the ribosome; RNC, ribosomenascent chain complex; SRP, signal recognition particle; $T F$, trigger factor.

presence of a signal sequence. O $\mathrm{n}$ targeting of the RN C-SRP complex to the ER for co-translational membrane insertion, the L23a/L35 site is one of the main connections between the ribosome and the translocon (Beckmann et al., 2001; Morgan et al., 2002). As the interactions of L23a/L35 with SRP54 and the Sec translocon seem to be mutually exclusive, SRP54 must be released from L23a/L35 before the establishment of the ribosome-translocon junction. The SRP receptor in the ER membrane might be involved in this step, as it triggers the evacuation of SRP5 4 from L23a (Pool et al., 2002).

In E. coli, L23 (a homologue of L23a) seems to have an even more intricate role. It functions as an attachment site not only for the SRP (Gu et al., 2003; U llers et al., 2003), but also for the chaperone and prolyl isomerase trigger factor (TF; Kramer et al., 2002). In addition, TF has been shown to be associated with L29 (a homologue of L35). The role of TF in protein targeting and folding is unclear. Crosslinking studies have identified TF as the first chaperone to interact generically with nascent polypeptides (Hesterkamp et al., 1996; Valent et al., 1995), and recent evidence points to its cooperation with another chaperone, D naK, in co-translational folding (D euerling et al., 1999, 2003; Teter et al., 1999). It has been suggested that TF also has a discriminatory role in targeting by interacting specifically with the early mature region of secretory proteins, thereby reducing the affinity of their mildly hydrophobic signal peptide for the SRP (Beck et al., 2000). This would select secretory proteins for subsequent interaction with the targeting factor $\operatorname{Sec} B$, which delivers them to SecA at the Sec translocon by a mechanism that is essentially post-translational. However, other studies suggest that TF binds to the nascent chain by default and that only the SRP can confer targeting specificity because of its high affinity for newly synthesized, strongly hydrophobic targeting sequences in nascent IMPS (Lee \& Bernstein, 2001; Valent et al., 1997). In either of these mechanisms, L23 might coordinate the positioning of TF and SRP on the ribosome, perhaps in response to specific structural features of the synthesized polypeptide.

Interestingly, it has recently been shown that the eukaryotic SRP has a higher affinity for active, translating ribosomes than for those that are inactive, even when the nascent chain is still entirely located in the ribosomal exit tunnel (Flanagan et al., 2003). Possibly, the peptide that is buried in the exit tunnel causes a change in conformation of the $L 23$ region on the ribosome surface, thereby influencing its affinity for SRP and TF. In this scenario, the function of TF might be to prevent the inadvertent association of ribosomes that do not carry a nascent IMP with the Sec translocon by covering the L23/L29 component of the ribosome-translocon junction site. In support of this idea, inactivation of the TF gene has been shown to accelerate the translocation of secretory proteins and to suppress their dependence on $\mathrm{Sec} B$, whereas overproduction of TF retards their translocation (Lee \& Bernstein, 2002).

\section{The Sec translocon}

Before discussing the role of YidC in IM P assembly it is necessary to give a more detailed introduction to the Sec translocon. Its core consists of the integral IMPs SecY, SecE and SecG, and the peripheral subunit SecA. Together, SecY, SecE and SecG form a proteinconducting channel, and the peripheral subunit SecA pushes proteins into and through this channel (Manting \& Driessen, 2000). SecA is not required for SRP-mediated targeting and the initial membrane insertion of a nascent IM P (Scotti et al., 1999), but is necessary for the translocation of large periplasmic loops (Andersson $\&$ von Heijne, 1993; Fröderberg et al., 2003).

The higher order oligomeric form of the Sec translocon is a matter of debate. It is clear that SecY, SecE and SecG form a heterotrimer (Manting \& D riessen, 2000). Electron microscopic examination of purified translocons indicates that dimeric SecA catalyses the assembly of four SecYEG heterotrimers into what might be one large protein-conducting channel (Manting \& Driessen, 2000). However, fluorescence resonance energy transfer analysis of SecYE heterodimers suggests that they form constitutive oligomers (M ori et al., 2003). Further data, from twodimensional crystallography, analytical ultracentrifugation and native-gel electrophoresis experiments, have led to the recent proposal that the active $\mathrm{Sec}$ translocon is comprised of two SecYEG heterotrimers (Bessonneau et al., 2002; Breyton et al., 2002). However, it should be noted that SecA was not present in the Sec-translocon preparations used in this last set of experiments. Finally, co-immunoprecipitation and crosslinking experiments indicate that even single SecYEG heterotrimers can form protein-conducting channels (Yahr \& Wickner, 2000). H owever, this last study does not exclude the possibility that the SecYEG 
heterotrimers form higher order oligomers. In other words, protein-conducting channels could be grouped in the membrane and this might be necessary in particular situations, such as for the assembly of IM P complexes.

The oligomeric states of other components of the complex are less debated. SecA itself forms stable dimers, which may dissociate during protein translocation (Or et al., 2002). SecD, SecF and YajC are nonessential Sec-translocon subunits that facilitate the translocation reaction and form an auxiliary subcomplex that can be copurified with the SecYEG complex under certain conditions. Whether this SecDFYajC complex has any role in IM P insertion and/or assembly is not yet clear (De G ier \& Luirink, 2001). It is known that during IMP assembly, TMs are arrested in the Sec translocon and subsequently move out into the lipid bilayer (Chen et al., 2002; De Gier \& Luirink, 2001), and the protein YidC is thought to assist in this transfer (D e Gier \& Luirink, 2001).

YidC and assembly of SRP/Sec-translocon-dependent IMPS YidC is a relatively abundant IMP that exists in two physical states: one that is associated with the Sec translocon and another that is not (Chen et al., 2002; De Gier \& Luirink, 2001). The Secassociated form has been implicated in the integration of IMPS that are targeted through the SRP pathway to the Sec translocon (Fig. 1C). Depletion of YidC has a relatively mild effect on the integration of this class of proteins into the membrane as judged by the accessibility of their periplasmic domains to proteases (for example, see Fröderberg et al., 2003; Samuelson et al., 2000; U rbanus et al., 2001). These data indicate that YidC influences the efficiency and kinetics of Sec-dependent membrane integration without being essential for this process.

Little is known about the oligomeric organization of YidCcontaining Sec translocons. In vitro reconstitution and crosslinking studies indicate that YidC and SecYEG are in close proximity, at least during the initial stages of IMP insertion (Urbanus et al., 2001; van der Laan et al., 2001; Beck et al., 2001; Houben et al., 2002). Consistent with these observations, YidC can be copurified with the core SecYEG translocon, and overexpression of Sectranslocon components positively affects $Y i d C$ expression, which suggests a functional relationship (Scotti et al., 2000). In addition, it has been shown that the chloroplast homologue of YidC, Albino3 (Alb3), interacts directly with the chloroplast homologue of SecY, cpSecY (Klostermann et al., 2002). These observations indicate that YidC interacts with SecYEG, possibly through the SecY subunit, although it has also been reported that YidC interacts with SecD and SecF to form a heterotetrameric SecDFYajCYidC accessory complex (N ouwen \& D riessen, 2002). W hether the association of YidC with the Sec translocon is static or dynamic is an issue that remains to be addressed. If the process is dynamic, YidC might be recruited from the 'free' pool on demand.

The function of YidC in Sec-dependent IMP integration is uncertain. In vitro crosslinking studies to monitor the molecular environment of nascent IMPS- 'integration intermediates'- revealed prominent contacts with YidC (for example, see Beck et al., 2001; Scotti et al., 2000; U rbanus et al., 2001). These contacts are specific to TM regions and are made only in the context of the translating ribosome. Hence, it has been suggested that YidC facilitates the partitioning of TM segments into the lipid bilayer after their co-translational insertion into the Sec translocon (Scotti et al., 2000). Interestingly, YidC was found to be crosslinked to differentTM s in the same nascent polytopic IMP M tIA (Beck et al., 2001). This observation may reflect a role for YidC in the packing and assembly of TM s in or near the Sec translocon before their coordinated release into the lipid bilayer.

Crosslinking analysis of nascent IM Ps of differing lengths that are derived from the bitopic IMP FtsQ showed that its TM first contacts SecY and then YidC (U rbanus et al., 2001). H ow ever, in a more extensive study using the polytopic model IMP Lep, different segments in the firstTM could be crosslinked to both SecY and YidC, even when the nascent chains were very short and the first TM was not yet fully exposed outside the ribosome (Houben et al., 2002). This suggests that YidC also has an early role in the reception and recognition of TMs, at a stage when the translating ribosome must be in close proximity to the Sec translocon. It is possible thatYidC assists in the docking of specifically those ribosomes that synthesize IM Ps to allow co-translational insertion.

It should be noted that only a few model IMPs were used in these studies. Clearly, more information is necessary to assess fully the functions of YidC in the insertion and assembly of Sec-dependent IM Ps of different complexity and topology.

\section{YidC and assembly of Sec-translocon-independent IM Ps}

W hereas YidC depletion has only a moderate effect on the assembly of SRP/Sec-translocon-dependent IM Ps, it almost completely blocks the integration of small phage-coat proteins that require neither the SRP pathway nor the Sec translocon for insertion into the membrane (for the pathway, see Fig. $1 \mathrm{~A}$ ). Nothing is known about the Sec-independent structure of YidC, but the homologue of this protein in the fungus $\mathrm{N}$ eurospora crassa, which has neither an SRP pathway nor a Sec translocon, forms homo-oligomeric complexes that are thought to constitute translocons (Nargang et al., 2002). For YidC, it will be crucial to determine whether it functions in a homo- or a hetero-oligomeric complex during the assembly of SRP/Sec-independent phage-coat proteins.

Considering that $Y i d C$ is not required for the assembly of the SRP/Sec-dependent IMPS (many of which are essential cellular proteins), and that phage-coat proteins, which do require YidC, are obviously not essential for growth, why is YidC essential for viability? A recent study has shown that the loss of YidC rapidly leads to defects in the functional assembly of cytochrome o oxidase and the $\mathrm{F}_{0} \mathrm{~F}_{1}$-ATPase complex, which is reminiscent of the effects of mutations in the yidC homologue OXA1 in the yeast mitochondrial inner membrane (van der Laan et al., 2003). Specifically, the integration of CyoA (subunit II of the cytochrome 0 oxidase) and $F_{0} C$ (membrane subunit of the $F_{0} F_{1}$-ATPase) into their respective complexes are exceptionally sensitive to the depletion of YidC. Both of these proteins are rather small integral membrane proteins with two closely spaced TMs, and are components of multisubunit oligomeric complexes. It is therefore possible that they are natural substrates of the YidC-dependent/ Sec-independent membrane protein insertion pathway that is also used by the phage-coat proteins in E. coli. Alternatively, it is possible that YidC is involved in the assembly of IM P complexes, as has been suggested for its mitochondrial homologue 0 xal (Stuart, 2002). Therefore, the decrease of CyoA and $\mathrm{F}_{0} \mathrm{C}$ on YidC depletion could also be explained by the rapid degradation of CyoA molecules that are not part of cytochrome o oxidase complexes, and of $\mathrm{F}_{0} \mathrm{C}$ molecules that are not part of $\mathrm{F}_{0} \mathrm{~F}_{1}$-ATPase complexes. 


\begin{abstract}
A third IMP assembly pathway in Escherichia coli?
Membrane protein assembly studies in chloroplasts gave the first hints towards a possible third IMP assembly pathway in E. coli (Fig. 1B). In chloroplasts, the cpSRP/Alb3 pathway is involved in the assembly of many thylakoid membrane proteins (Eichacker \& Henry, 2001), and CPSRP and Alb3 are the chloroplast homologues of E. coli SRP and YidC, respectively. Recently, it was also shown that, in E. coli, engineered model IM Ps were assembled into the inner membrane in an SRP/YidC-dependent and Sec-translocon-independent fashion (Fröderberg et al., 2003). It is thus conceivable that an SRP/YidC pathway similar to the cPSRP/Alb3 pathway in chloroplasts, is operational in E. coli.
\end{abstract}

\section{Conclusions and perspectives}

In E. coli, the large ribosomal subunit component $L 23$ constitutes an overlapping binding site for TF and SRP near the nascentchain exit site (Fig. 1). We propose that, by virtue of this property, the ribosome might have a more active role in the selection of targeting pathways than had previously been anticipated. Future binding studies are likely to reveal how the binding of TF and SRP to the ribosome is regulated and how this influences the interactions of these factors with the nascent substrate. Decisions that are made at this stage will also affect downstream stages in the targeting process and so not only the binding of SRP and TF to, but also their release from, the ribosome is of interest. Indeed, it seems likely, on the basis of extrapolation of structural data from the ribosome-translocon junction in yeast (M organ et al., 2002), that L23 must be cleared of binding partners to allow its direct interaction with the Sec translocon during co-translational membrane insertion. At this stage, YidC is near the ribosome-associated SRP/Sec-dependent IM P, but whether it has any role in ribosome docking remains to be investigated. A function for YidC in the reception of ribosomes that synthesize Sec-independent IMPs, as anticipated for the mitochondrial YidC homologue Oxal (Hell et al., 2001), is an interesting possibility that warrants further investigation.

In E. coli there are at least two IMP assembly pathways, the SRP-Sec-translocon-YidC pathway and the YidC pathway, in which the role of YidC is distinct. In addition, a third IMP assembly pathway, the SRP-YidC pathway, may be operational in this organism (Fig. 1). Some insight into the substrates of the targeting and assembly pathways in which YidC is involved has been obtained. However, the main challenges for the future will be to establish how this protein operates in different complexes so that it executes various tasks in IMP assembly, exactly what these tasks are and how they relate to the structural characteristics of YidC. In a general sense, YidC may be considered as an integral membrane chaperone that interacts transiently with membrane proteins during their biogenesis and stimulates their correct assembly. More specific functions have been proposed on the basis of limited experimental data of a mainly biochemical nature. YidC might facilitate the lipid partitioning of TM segments of Sec-independent IMPS (without being absolutely essential for this process) and stimulate the translocation of their periplasmic domains (Samuelson et al., 2001). For Sec-dependent IM Ps, YidC might recognize individual TM $s$ in the context of the Sec translocon early during biogenesis and facilitate their folding and, finally, their partitioning into the lipids (De Gier \& Luirink, 2001; Houben et al., 2002). YidC might also collect together different
TM s of polytopic IM Ps to catalyse their assembly and coordinated release into the lipid bilayer (Beck et al., 2001). As mentioned before, YidC might even function in the assembly of oligomeric IM P complexes. Finally, YidC might also have a role in the quality control of IMPs, although there is no evidence as yet for a link with proteolytic enzymes.

It is clear that a full appreciation of the versatile roles of both the ribosome and YidC in IMP biogenesis in E. coli will require a combined biochemical, genetic and structural effort.

\section{ACKN O W LED GEM ENTS}

Wethank G. von Heijneand K.-J. van Wijk for critically reading the manuscript. J.-W.d.G. is a recipient of an EM BO Young Investigator award.

\section{REFERENCES}

Andersson, H. \& von Heijne, G. (1993) Sec dependent and sec independent assembly of E. coli inner membrane proteins: the topological rules depend on chain length. EM BO J., 12, 683-691.

Avdeeva, O.N., M yasnikov, A.G., Sergiev, P.V., Bogdanov, A.A. Brimacombe, R. \& Dontsova, O.A. (2002) Construction of the 'minimal' SRP that interacts with the translating ribosome but not with specific membrane receptors in Escherichia coli. FEBS Lett., 514, 70-73.

Beck, K., Wu, L.F., Brunner, J. \& Muller, M . (2000) Discrimination between SRP- and SecA/SecB-dependent substrates involves selective recognition of nascent chains by SRP and trigger factor. EM BO J., 19, 134-143.

Beck, K., Eisner, G., Trescher, D., Dalbey, R.E., Brunner, J. \& Muller, M. (2001)YidC, an assembly site for polytopic Escherichia coli membrane proteins located in immediate proximity to the SecYE translocon and lipids. EMBO Rep., 2, 709-714.

Beckmann, R., Spahn, C.M., Eswar, N., Helmers, J., Penczek, P.A., Sali, A., Frank, J. \& Blobel, G. (2001) Architecture of the protein-conducting channel associated with the translating 80 S ribosome. Cell, 107, 361-372.

Bessonneau, P., Besson, V., Collinson, I. \& Duong, F. (2002) The SecYEG preprotein translocation channel is a conformationally dynamic and dimeric structure. EMBO J., 21, 995-1003.

Breyton, C., Haase, W., Rapoport, T., Kuhlbrandt, W. \& Collinson, I. (2002) Three-dimensional structure of the bacterial protein-translocation complex SecYEG. N ature, 418, 662-665.

Chen, M., Xie, K., Jiang, F., Yi, L. \& Dalbey, R.E. (2002)YidC, a newly defined evolutionarily conserved protein, mediates membrane protein assembly in bacteria. Biol. Chem., 383, 1565-1572.

De Gier, J.W. \& Luirink, J. (2001) Biogenesis of inner membrane proteins in Escherichia coli. Mol. Microbiol., 40, 314-322.

Deuerling, E., Schulze-Specking, A., Tomoyasu, T., Mogk, A. \& Bukau, B. (1999) Trigger factor and D naK cooperate in folding of newly synthesized proteins. N ature, 400, 693-696.

Deuerling, E., Patzelt, H., Vorderwulbecke, S., Rauch, T., Kramer, G., Schaffitzel, E., Mogk, A., Schulze-Specking, A., Langen, H. \& Bukau, B. (2003) Trigger factor and D naK possess overlapping substrate pools and binding specificities. Mol. M icrobiol., 47, 1317-1328.

Eichacker, L.A. \& Henry, R. (2001) Function of a chloroplast SRP in thylakoid protein export. Biochim. Biophys. Acta, 1541, 120-134

Flanagan, J.J., Chen, J.C., M iao, Y., Shao, Y., Lin, J., Bock, P.E. \& Johnson, A.E. (2003) SRP binds to ribosome-bound signal sequences with fluorescence-detected subnanomolar affinity that does not diminish as the nascent chain lengthens. J. Biol. Chem., 278, 18628-18637.

Fröderberg, L., H ouben, E., Samuelson, J., Chen, M., Park, S., Phillips, G.J., Luirink, J., D albey, R.E. \& de Gier, J.W.L. (2003) Versatility of inner membrane protein biogenesis in Escherichia coli. Mol. M icrobiol., 47, 1015-1027.

Gu, S.Q., Peske, F., W ieden, H.J., Rodnina, M.V. \& W intermeyer, W. (2003) The signal recognition particle binds to protein $L 23$ at the peptide exit of the Escherichia coli ribosome. RNA, 9, 566-573.

Hell, K., N eupert, W. \& Stuart, R.A. (2001) 0 xalp acts as a general membrane insertion machinery for proteins encoded by mitochondrial DNA. EMBO J., 20, 1281-1288.

Herskovits, A.A., Bochkareva, E.S. \& Bibi, E. (2000) New prospects in studying the bacterial signal recognition particle. Mol. M icrobiol., 38, 927-937. 
Herskovits, A.A. et al. (2001) Evidence for coupling of membrane targeting and function of the signal recognition particle (SRP) receptor FtsY. EMBO Rep., 2, 1040-1046.

Hesterkamp, T., H auser, S., Lutcke, H. \& Bukau, B. (1996) Escherichia coli trigger factor is a prolyl isomerase that associates with nascent polypeptide chains. Proc. Natl Acad. Sci. USA, 93, 4437-4441.

Houben, E.N ., U rbanus, M .L., van der Laan, M ., ten Hagen-Jongman, C.M., D riessen, A.J., Brunner, J., O udega, B. \& Luirink, J. (2002)YidC and SecY mediate membrane insertion of a type I transmembrane domain. J. Biol. Chem., 277, 35880-35886.

Klostermann, E., D roste G en Helling, I., Carde, J.P. \& Schunemann, D. (2002) The thylakoid membrane protein ALB3 associates with the cpSecY-translocase in Arabidopsis thaliana. Biochem. J., 368, 777-781.

Kramer, G., Rauch, T., Rist, W., Vorderwulbecke, S., Patzelt, H., SchulzeSpecking, A., Ban, N., Deuerling, E. \& Bukau, B. (2002) L23 protein functions as a chaperone docking site on the ribosome. $N$ ature, 419 , 171-174.

Lee, H.C. \& Bernstein, H.D. (2001) The targeting pathway of Escherichia coli presecretory and integral membrane proteins is specified by the hydrophobicity of the targeting signal. Proc. N atl Acad. Sci. U SA, 98, 3471-3476.

Lee, H.C. \& Bernstein, H.D. (2002) Trigger factor retards protein export in Escherichia coli. J. Biol. Chem., 277, 43527-43535.

Manting, E.H . \& D riessen, A.J. (2000) Escherichia coli translocase: the unravelling of a molecular machine. Mol. Microbiol., 37, 226-238.

Morgan, D.G., Menetret, J.F., N euhof, A., Rapoport, T.A. \& Akey, C.W. (2002) Structure of the mammalian ribosome-channel complex at 17A resolution. J. Mol. Biol., 324, 871-886.

M ori, H., Tsukazaki, T., Masui, R., Kuramitsu, S., Yokoyama, S., Johnson, A.E., Kimura, Y., A kiyama, Y. \& Ito, K. (2003) Fluorescence resonance energy transfer analysis of protein translocase. SecYE from Thermus thermophilus H B8 forms a constitutive oligomer in membranes. J. Biol. Chem., 278, 14257-14264.

N argang, F.E., Preuss, M ., N eupert, W. \& Herrmann, J.M . (2002) The 0 xal protein forms a homooligomeric complex and is an essential part of the mitochondrial export translocase in N eurospora crassa. J. Biol. Chem., 277, 12846-12853.

Nouwen, N . \& D riessen, A.J. (2002) SecD FyajC forms a heterotetrameric complex with YidC. Mol. Microbiol., 44, 1397-1405.

O r, E., Navon, A. \& Rapoport, T. (2002) Dissociation of the dimeric SecA ATPase during protein translocation across the bacterial membrane. EMBO J., 21, 4470-4479.

Pool, M .R., Stumm, J., Fulga, T.A., Sinning, I. \& D obberstein, B. (2002) Distinct modes of signal recognition particle interaction with the ribosome. Science, 239, 1345-1348.

Prinz, A., Behrens, C., Rapoport, T.A., Hartmann, E. \& Kalies, K.U. (2000) Evolutionarily conserved binding of ribosomes to the translocation channel via the large ribosomal RNA. EMBO J., 19, 1900-1906.

Samuelson, J.C., Chen, M., Jiang, F., M oller, I., W iedmann, M., Kuhn, A., Phillips, G.J. \& Dalbey, R.E. (2000)YidC mediates membrane protein insertion in bacteria. $N$ ature, 406, 637-641.

Samuelson, J.C., Jiang, F., Yi, L., Chen, M., de Gier, J.W., Kuhn, A. \& Dalbey, R.E. (2001) Function of YidC for the insertion of M 13 procoat protein in Escherichia coli: translocation of mutants that show differences in their membrane potential dependence and Sec requirement. J. Biol. Chem., 276, 34847-34852.

Scotti, P.A., Valent, Q .A., M anting, E.H., U rbanus, M.L., D riessen, A.J., O udega, B. \& Luirink, J. (1999) SecA is not required for signal recognition particle-mediated targeting and initial membrane insertion of a nascent inner membrane protein. J. Biol. Chem., 274 29883-29888.
Scotti, P.A., U rbanus, M .L., Brunner, J., de Gier, J.W., von Heijne, G ., van der Does, C., Driessen, A.J., O udega, B. \& Luirink, J. (2000)YidC, the Escherichia coli homologue of mitochondrial $0 \times a 1 p$, is a component of the Sec translocase. EM BO J., 19, 542-549.

Stuart, R. (2002) Insertion of proteins into the inner membrane of mitochondria: the role of the 0 xal complex. Biochim. Biophys. Acta, 1592, 79-87.

Teter, S.A., H oury, W.A., Ang, D., Tradler, T., Rockabrand, D., Fischer, G., Blum, P., G eorgopoulos, C. \& H artl, F.U. (1999) Polypeptide flux through bacterial H sp70: D naK cooperates with trigger factor in chaperoning nascent chains. Cell, 97, 755-765.

Ullers, R.S., H ouben, E.N.G., Raine, A., ten Hagen-Jongman, C.M ., Ehrmann, M ., O udega, B., Harms, N. \& Luirink, J. (2003) Interplay of SRP and Trigger Factor at L23 near the nascent chain exit site on the E. coli ribosome. J. Cell Biol., 161, 679-684.

U rbanus, M.L., Scotti, P.A., Froderberg, L., Saaf, A., de Gier, J.W., Brunner, J., Samuelson, J.C., D albey, R.E., O udega, B. \& Luirink, J. (2001) Secdependent membrane protein insertion: sequential interaction of nascent FtsQ with SecY and YidC. EM BO Rep., 2, 524-529.

Valent, Q.A., Kendall, D.A., High, S., Kusters, R., O udega, B. \& Luirink, J. (1995) Early events in preprotein recognition in E. coli: interaction of SRP and trigger factor with nascent polypeptides. EM BO J., 14, 5494-5505.

Valent, Q.A., de Gier, J.W.L., von H eijne, G., Kendall, D.A., ten HagenJongman, C.M ., O udega, B. \& Luirink, J. (1997) Association of nascent membrane and presecretory proteins with Signal Recognition Particle and Trigger Factor. Mol. Microbiol., 25, 53-64.

van der Laan, M., H ouben, E.N., N ouwen, N ., Luirink, J. \& D riessen, A.J. (2001) Reconstitution of Sec-dependent membrane protein insertion: nascent FtsQ interacts with YidC in a SecYEG -dependent manner. EMBO Rep., 2, 519-523.

van der Laan, M., U rbanus, M.L., ten Hagen-Jongman, C.M ., N ouwen, N ., O udega, B., Harms, N., D riessen, A.J. \& Luirink, J. (2003) A conserved fuction of YidC in the biogenesis of respiratory complexes. Proc. $N$ atl Acad. Sci. USA, 100, 5801-5806.

Yahr, T.L. \& Wickner, W.T. (2000) Evaluating the oligomeric state of SecYEG in preprotein translocase. EM BO J., 19, 4393-4401.

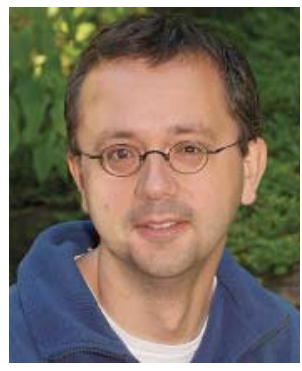

\section{Jan-Willem L. deGier istherecipient of an EM BO Young Investigator Award}

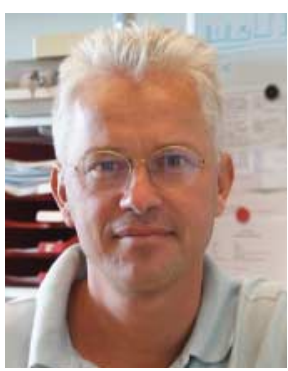

Joen Luirink 